\title{
Assessing the Impact of Cafeteria Food Related Problems on the Academic Achievement of Students in Debre Berhan University
}

\author{
Bezarede Mekonnen ${ }^{1, *}$, Tadesse Ayele ${ }^{2}$ \\ ${ }^{1}$ Department of Statistics, College of Natural and Computational Science, Debre Berhan University, Debre Berhan, Ethiopia \\ ${ }^{2}$ Department of Mathematics, College of Natural and Computational Science, Debre Berhan University, Debre Berhan, Ethiopia
}

Email address:

bezaredemekonnen70@gmail.com(B. Mekonnen), tadesseayele02@gmail.com (T. Ayele)

${ }^{*}$ Corresponding author

\section{To cite this article:}

Bezarede Mekonnen, Tadesse Ayele. Assessing the Impact of Cafeteria Food Related Problems on the Academic Achievement of Students in Debre Berhan University. American Journal of Theoretical and Applied Statistics. Vol. 9, No. 2, 2020, pp. 21-26.

doi: $10.11648 /$ j.ajtas.20200902.12

Received: October 9, 2019; Accepted: April 15, 2020; Published: April 28, 2020

\begin{abstract}
Education is a process through which mankind transmit experience, new findings and value accumulate over time with the aim of individuals and societies, to make all around participation in the development process. Sufficient healthy intake of food is essential to brain function. Moreover, maximizing brain function is a prime factor in seizing appropriate cognitive capability for example, ability to focus, comprehension, evaluation, and application in learning. Therefore, food is advisable take account for quality and stuffiness of food to protect our students from different infection and declining on their academic performance. The study was trying to assess the impact of Debre Berhan university cafeteria food related problems on the academic achievement of students. The general objective of the study is to analyze statistically the impact of food related problems on academic achievement of students. The study used well organized questionnaire and the source of data is primary data which will done by direct contact of respondents and secondary data which will be obtained from the registrar of Debre Berhan university. The collected data is analyzed by using descriptive and inferential statistics. The sample size determination is made by using statistical formula. Descriptive statistics and multiple linear regression analysis is applied to examine the relationship between the dependent variable academic achievement and food related problem independent variables. Multiple linear regression model is a mathematical equation that provides prediction of the value of dependent variable based on known value of independent variables. The analysis of multiple linear regression revealed that the academic achievement of students was mostly affected by adequacy of food supply, quality of cafeteria sanitation, health related problem, food sanitation and personal hygiene of cafeteria workers, time of cafeteria and those factors affect the academic achievement of students.
\end{abstract}

Keywords: Education, Food Related Problem, Academic Achievement and Multiple Linear Regression

\section{Introduction}

Education is the process by people can acquire the knowledge habits, values and attitude. It enables people to become more productive member of the society and which is also a man transmits his experiences, new findings and values accumulated over the years. In his/her struggle for survival and development through generation. Therefore, education plays a great role in the development and socioeconomic welfare of the society. In many developing countries peoples are unable to get an adequate nutrition to survive their living standards. One of the problems is that; food problem that is exist in developing countries [1].

The concept of food is anything that can fit for human being for the essential purpose, like protecting, building and gain energy. In the university the students do not worry about what they are eating because the government allocates budget for food service to the university students. To provide this service interested to study the conditions and impact of food problem at cafe which gives service to the students. It is 
advisable to take account the quality and stuffiness of the students to protect our students' from different infection and declining on their academic achievement to do this everybody would take the responsibility because of food is one of our basic needs. In general, food is defined as any substance that can people eat and drink to achieve adequate nutrition. This means that people maintain life, physical, cognitive and social development. Therefore, food has to meet physiological requirements in terms of quantity and quality to be socially and culturally acceptable [2].

According to an acceptable definition food security is to access all people times to enough food for living with an active healthy life. If food security is achieved, an adequate food quality or quantity may have socio-cultural acceptability is available, accessed and satisfactorily utilized by all individuals at all times to achieve good nutrition for health and happy life. Therefore, food is advisable take account for quality and stuffiness of food to protect our students from different infection and declining on their academic performance [3].

Our investigation is to attain the educational goal one does can should have to take account and settle the problems that affects the student's academic achievements of the desired goal. These factors can be known and get solutions through conduction of educational research. Various studies have been conducted to analyze the impact of food related problems on the student's academic achievement. However this study attempts to look or to investigate due to food related problem with different factors that affect the student's academic achievement in Debre Berhan University. The general objective of this study is assessing the impact of food related problems on the academic achievement of the students in Debre Berhan University.

\section{Literature Review}

Food is any substance that can fit for human being for essential purpose, like protecting, building and gain energy and also defined as anything that people eat and drink to achieve an adequate nutrition. Status meaning that maintain life and physical, cognitive and social development [2].

Right to food is UN charter every signatory government is, obligated to provide adequate food to all citizens. Mankind has made remarkable progress in the twentieth century. More people than ever before live longer are healthier and eat betterand more nutritious food. Yet, by the most recent FAO estimate about 790 million persons are food insecure and the SCN estimate about 150 million children suffer chronic under nutrition [4].

Now, we understood, though not fully that both neighborhood socio-economic environment and individual educational status may be associated with self-reported poor health and poor health behaviors. As mentioned previously it has been found that those living on a low income or in socially advantaged area may face higher food prices [5].

It should be noted that this is not a consistent finding found that low income areas have three times the density of fast food outlets per head population data. Furthermore, the phenomenon for supersizing. That is offering larger food portions for little increase in price for foods eaten outside home provides in expensive extra energy at lower unit cost.

A Preliminary Examination of the Factors of food related problems, like: nutritional neuroscience, malnutrition, diet adequacy, low food supply, low quality of food sanitation and low quality of cafeteria sanitation have great impacts on the academic performance.

Sufficient healthy intake of food is essential to brain function [6]. Moreover, maximizing brain function is a prime factor in seizing appropriate cognitive capability - for example, ability to focus, comprehension, evaluation, and application - in learning.

Kretchmer addresses three aspects related to the foodlearning relationship, including offering.

1) The nutritional needs of the brain by analyzing research from cognitive neuroscience and healthcare fields.

2) An exploration malnutrition in terms of both over and under-nutrition, and a discussion of the implications of malnutrition at different stages in development.

3) A consideration of the effect of diet quality on academic achievement.

Finally, a synthesis of these three aspects, a discussion of related learning theory and current debate, and practical implications for educational settings is tendered. Nutrition is the central variable in this discussion, so a basic understanding of several dietetic terms is essential [7].

Malnutrition is an imbalance between nutrients the body needs and those the body receives. Malnutrition can exist as undernutrition, which is a general and long-term deficiency of calories, or as its opposite, overnutrition. Though people

experiencing overnutrition consume more than enough calories, they are often deficient in various nutrients, due often to diets that are comprised of nutrient-poor and energydense foods such as sugary soft drinks and fast-food items [8].

Finally, nutritional neuroscience is the study of the effects of food products on behavior and brain function [9].

Paucity of Research on the Effects of Food in Learning

Most might share these theoretical assumptions as a starting point for the relationship of food and learning:

1) that consistent consumption of an adequate, highquality diet improves academic performance;

2) that a higher-quality diet corresponds to higher academic performance and

3) that malnutrition contributes to the low levels of academic performance prevalent among person living in poverty.

Yet, while there may be a consensus that food is essential to learning, there is little empirical research that examines the exact relationship between student's overall diet and academic achievement. Neither are the results conclusive in determining the precise nature and degree of the foodlearning relationship [10]. Controversy exists regarding the specific foods that comprise an adequate diet, the effect of food intake throughout development on immediate and ensuring academic outcomes, and the nature and extent of the 
relationship between food and academic performance. The controversy seems to exist because of divergent conclusions that have emerged from various studies. Some of these studies may have such mixed results because of the research design difficulties.

Another likely explanation for lack of concrete knowledge about the causal effects of nutrition on the brain and academic achievement is that designing randomized control trials (RCTs) to study the effects of specific nutritional aspects is difficult [11]. Many RCTs either are too short in duration, too narrow in focus, or too small in sample size, or utilize measurement tools that are too unreliable and subjective to generalize about nutrition's long-term effects on broad populations.

Studies using other methods often have complex confounding variables that compromise the validity of the study's results. Statistical manipulation is necessary to control for extraneous variables, which maintains internal validity, but often shifts the study's conclusions from authentic correlations to theoretical inferences [12]. Thus, while a controversy persists in some quarters of the debate of food and academic performance, we tend to take the side of sound conclusions from a number of studies which demonstrate the positive correlation of healthy food intake and increased academic performance. These conclusions guide the remainder of this article.

1) The Relationship of Food and Academic Performance Published by DigitalCommons@Liberty University, 2012 and varied meals accessible to children [13].

Even with data suggesting a correlation between nutrition and academic performance, schools may justifiably feel uncomfortable and unqualified to recommend dietary changes. Additional research might be useful to further resolve the divided opinions about diets and food intake [13].

Food is essential to academic performance because it provides the energy necessary for cognition. Improving persons's diet quality by increasing their consumption of fruits, vegetables, and whole grains, and reducing their intake of saturated fats and extra-calorie foods will provide more adequate energy and nutrients [14].

\section{Methodology}

\subsection{Study Area}

This study is conducted Assessing the impact of food related problems on the academic achievement of the students in Debre Berhan University. Debre Berhan University is found in Debre Berhan town which is found in north Shewa zone in Amhara region. Debre Berhan is located at $130 \mathrm{~km}$ north of Addis Ababa which is the capital city of Ethiopia.

\subsection{Study Variables}

In this study the dependent variable and independent variables are:

Dependent Variable: Academic achievement of student (Y)
Independent Variables:
a. Adequacy of food supply
b. quality of cafeteria sanitation
c. Personal hygiene of cafeteria workers
d. food sanitation
e. time of cafeteria
f. unbalanced cost giving for non-cafeteria students
g. health related problem with related to food insecurity

\subsection{Linear Regression Model}

The regression model is a mathematical equation that provides prediction of the value of dependent variable based on known value of one independent variable. The generalized model for the linear regression models is:-

$$
\mathrm{Y}_{\mathrm{i}}=\beta_{0}+\beta_{1} \mathrm{X}_{1}+\mathrm{C}_{\mathrm{i}}
$$

Where, $\mathrm{Y}_{\mathrm{i}}=$ dependent variable

$\beta_{0}=$ constant

$\mathrm{X}_{1}=$ independent variable

$\mathrm{B}_{1}=$ parameter to be estimated

$\mathrm{Ei}=$ errors terms or other factors not included in this study

Multiple Linear Regression Model

The regression model is a mathematical equation that provides prediction of the value of dependent variable based on known value of independent variables. The generalized model for the multiple linear regression models is:-

$$
\mathrm{Y}_{\mathrm{i}}=\beta_{0}+\beta_{1} \mathrm{X}_{1}+\beta_{2} \mathrm{X}_{2}+\beta_{3} \mathrm{X}_{3}+\ldots+\beta_{\mathrm{k}} \mathrm{X}_{\mathrm{k}}+\mathrm{C}_{\mathrm{i}}
$$

Where, $\mathrm{Y}_{\mathrm{i}}=$ academic achievement of students

$\mathrm{X}_{\mathrm{k}}=\mathrm{k}^{\text {th }}$ factor (independent variable) that affect academic achievement of the students

$\beta_{\mathrm{k}}=\mathrm{k}^{\text {th }}$ population parameters to be estimated

$\mathrm{Ei}=$ errors terms or other factors not included in this study

Parameter Estimation of the Model

The model parameter is estimated by least square estimate method. That is, is the least square estimate of the parameters. is an unbiased estimate of $\beta$ and it is calculated as:-

By letting $Q(\beta)=C^{\prime} C$ where, $Q(\beta)$ is sum square of residual $\mathrm{C}=\mathrm{Y}$ - and $=\mathrm{X}$

Therefore $Q(\beta)=\left(Y_{-}\right)^{\prime}(Y-)=(Y-X)^{\prime}(Y-X)=Y^{\prime} Y-$ 2'X'Y+'X'X

$$
Q(\beta)=Y^{\prime} Y-2 X^{\prime} Y+X^{\prime} X
$$

Then taking partial derivative of Q () with respect to $\beta$ and equating to zero. Then we get the estimated value of $\beta$. That is $=\left(X^{1} X\right)^{-1} X^{1} Y$

Where, $Y$ is vector of observation of academic achievement

$\mathrm{X}$ is matrix of factors affecting the academic achievement

$\beta$ is vectors of the coefficients of the independent variables Assumption of Multiple Linear Regressions

The major assumption of the multiple linear regression models are:-

a. The true relationship between response variable (Y) and repressors' must be linear

b. The error terms are uncorrelated. That is, no serial 
correlation (serial independence of the Ei's. it also implies that the covariance error terms became zero. i.e. $\operatorname{cov}(\mathrm{Ci}, \mathrm{Cj})=0$, for $\mathrm{ij}$.

c. Errors are normally distributed. i.e. $\mathrm{Ci} \mathrm{N}\left(0, \sigma^{2}\right)$

d. Interdependence of $\mathrm{Ci}$ and $\mathrm{Xi}$. i.e. the covariance between error term and repressors' became zero.

e. No multicolinearity between independent variables. i.e. no exact or strong linear relationship between independent variables

\section{Testing Overall Significance}

The overall test aims at finding out whether the explanatory variables $\left(\mathrm{X}_{1}, \mathrm{X}_{2}, \ldots, \mathrm{X}_{\mathrm{k}}\right)$ do actually any significant influence on dependent variable $(\mathrm{Y})$. The test of the overall significance of regression can be tested the analysis of variance (ANOVA) technique. The following table summarizes the idea of ANOVA technique.

Analysis of variance (ANOVA)

Table 1. Shows the analysis of variance table.

\begin{tabular}{llll}
\hline Source of variation & Sum squares (SS) & Degrees of Freedom (DF) & Mean Squares (MS) \\
\hline Regression & SSR & P-1 & MSR \\
Residual & SSE & $n-p$ & MSE \\
Total & SST & $n-1$ & \\
\hline
\end{tabular}

Where, SSR = sum squares of regressors

$\mathrm{SSE}=$ Sum squares of errors

$\mathrm{MS}=$

$\mathrm{p}$ - refers to the number of estimated parameters

$\mathrm{n}$ - the number of observation

$p-1$ refers to degrees of freedom of the numerator

$\mathrm{n}-1$ refers to degrees of freedom of the denominator

Therefore, the overall test of significance implies that testing the null hypothesis against the alternative hypothesis. That is shown below;

$\mathrm{H}_{0}: \beta_{1}=\beta_{2}=\beta_{3}=\beta_{4}=\ldots=\beta_{\mathrm{k}}=0$

Against

$\mathrm{H}_{1}$ : 0 , for at least one $\mathrm{j}(\mathrm{j}=1,2, \ldots, \mathrm{k})$

The most important statistics in the analysis of variance table are the p-value (P), R2 and adjusted $\mathrm{R}^{2}$ values. Collectively, these values tell you if the level means are significantly different from each other and how well the model fits the data.

$\mathrm{R}^{2}$ and adjusted $\mathrm{R}^{2}$ measures of how well the model fits the data. These values can help you select the model with the best fit.

$\mathrm{R}^{2}$ describes the amount of variation in the observed response values that is explained by the predictor $(s) . R^{2}$ always increases with additional factors. For example, the best five-factor model will always have a higher $\mathrm{R}^{2}$ than the best three-predictor model. Therefore, $\mathrm{R}^{2}$ is most useful when comparing models of the same size.

$$
\mathrm{R}^{2}=\mathrm{SSR} / \mathrm{SST}
$$

Where $\mathrm{SSR}=$ sum square of regression

$\mathrm{SST}=$ sum square of total

Adjusted $R^{2}=1-\left(1-R^{2}\right) n-1 / n-k$

Adjusted $\mathrm{R}^{2}$ is a modified $\mathrm{R}^{2}$ that has been adjusted for the number of terms in the model. If you include unnecessary terms, $\mathrm{R}^{2}$ can be artificially high. Unlike $\mathrm{R}^{2}$, adjusted $\mathrm{R}^{2}$ may get smaller when you add terms to the model. Use adjusted $\mathrm{R}^{2}$ to compare models with different numbers of predictors.

Hypothesis testing about individual regression coefficients

We can test whether a particular variable $X_{1}, X_{2}, X_{3}$, - - -, $\mathrm{X}_{\mathrm{k}}$ is significant or not holding other variable constant. The ttest is used to test a hypothesis about any individual partial regression coefficient. The partial regression coefficient measures the change in the mean value of $\mathrm{Y}$ implies $\mathrm{E}\left(\mathrm{Y} / \mathrm{X}_{1}\right.$, $\left.X_{2},-\cdots, X_{k}\right)$, per unit change in $X_{1}$, holding $X_{2}, X_{3}, \ldots, X_{k}$ constant.

The test statistic $\mathrm{t}=(-) / \operatorname{Se}() \mathrm{t}_{\alpha / 2}(\mathrm{n}-\mathrm{k})$ where $\mathrm{j}=1,2, \ldots, \mathrm{k}$

This is the observed (or sample) the value of the $t$ ratio, which we compare with the theoretical value of $t$ obtainable from the t-table with n-k degrees of freedom.

Now let us postulate that

$\mathrm{H}_{0}:=0$

Against the alternative

$\mathrm{H}_{1}: 0$, for at least one $\mathrm{j}(\mathrm{j}=1,2, \ldots, \mathrm{k})$

If the computed $t$ value exceeds the critical $t$ value at the chosen level of significance, we may reject the null hypothesis; otherwise, we may accept it ( $\mathrm{j}$ is not significant at chosen level of significance and hence the corresponding regression does not appear to contribute to the explanation of the variation in response variable Y) [15].

\section{Results and Discussion}

Regression Analysis

Table 2. Model summary.

\begin{tabular}{lllll}
\hline \multicolumn{2}{l}{ Model Summary } & & \\
\hline Model & $\mathbf{R}$ & R Square & $\begin{array}{l}\text { Adjusted R } \\
\text { Square }\end{array}$ & $\begin{array}{l}\text { Std. Error of } \\
\text { the Estimate }\end{array}$ \\
\hline 1 & $.940^{\mathrm{a}}$ & .883 & .854 & .22100 \\
\hline
\end{tabular}

a. Predictors: (Constant), NEUTRALL, time of cafeteria, Adequacy of food supply, gastric, dis agree, personal hygene of cafeteria workers, cost giving for non cafe students, typhus, neutral, cafeteria sanitation, strong disagree, food sanitation, DISAGRE, health related problem, AGRE, agree, STRONG AGREE

b. Dependent Variable: Comulative GPA

Interpretation for the above model summary table

Adjusted R Square value 0.854 it means; about $85.4 \%$ of variability in the student's academic achievement is explained by the total variability of independent variables in the estimated regression and other non-mentioned about $15.4 \%$ of factors in the estimated regression are not 
explained.

Table 3. Summary results for analysis of variance (ANOVA) table.

\begin{tabular}{lllllll}
\hline ANOVA $^{\mathbf{b}}$ & & & & & \\
\hline \multirow{2}{*}{ Model } & $\begin{array}{l}\text { Sum of } \\
\text { Squares }\end{array}$ & df & $\begin{array}{l}\text { Mean } \\
\text { Square }\end{array}$ & F & Sig. \\
\hline \multirow{2}{*}{$\begin{array}{l}\text { Regression } \\
\text { Residual }\end{array}$} & 25.129 & 17 & 1.478 & 30.265 & $.000^{\mathrm{a}}$ \\
& Rotal & 3.321 & 68 & .049 & & \\
\hline
\end{tabular}

a. Predictors: (Constant), NEUTRALL, time of cafeteria, Adequacy of food supply, gastric, dis agree, personal hygene of cafeteria workers, cost giving for non cafe students, typhus, neutral, cafeteria sanitation, strong disagree, food sanitation, DISAGRE, health related problem, AGRE, agree, STRONG AGREE

b. Dependent Variable: Comulative GPA
Interpretation for the overall test

1. Based on the above analysis of variance table we have to test the overall test of the regression model the following procedures are important.

Stating of null and alternative hypothesis

$\mathrm{H}_{0}: \beta_{1}=\beta_{2}=\beta_{3}=\beta_{4}=\ldots=\beta_{6}=0$ it means all explanatory variables have no significant impact on fitted model.

$\mathrm{H}_{1}: 0$, for at least one $\mathrm{j}(\mathrm{j}=1,2, \ldots 6)$. It means for at least one $\beta_{\mathrm{j} \text { 's }}$ have a significant effects on the fitted model

From the above ANOVA table, $\mathrm{p}$ value is 0.00 . This is less than the level of significance (0.05). So as one can interpret that at $5 \%$ level of significant at least one has significant impact on the regression model. Thus the overall model is statistically significant for the data.

Table 4. Multiple regression model.

\begin{tabular}{|c|c|c|c|c|c|c|c|c|c|c|}
\hline \multicolumn{11}{|c|}{ Coefficients $^{\mathrm{a}}$} \\
\hline \multirow{2}{*}{\multicolumn{2}{|c|}{ Model }} & \multicolumn{2}{|c|}{$\begin{array}{l}\text { Unstandardized } \\
\text { Coefficients }\end{array}$} & \multirow{2}{*}{$\begin{array}{l}\text { Standardized } \\
\text { Coefficients } \\
\text { Beta }\end{array}$} & \multirow{2}{*}{$\mathbf{t}$} & \multirow{2}{*}{ Sig. } & \multicolumn{2}{|c|}{$\begin{array}{l}95 \% \text { Confidence } \\
\text { Interval for B }\end{array}$} & \multicolumn{2}{|c|}{$\begin{array}{l}\text { Collinearity } \\
\text { Statistics }\end{array}$} \\
\hline & & B & $\begin{array}{l}\text { Std. } \\
\text { Error }\end{array}$ & & & & $\begin{array}{l}\text { Lower } \\
\text { Bound } \\
\end{array}$ & $\begin{array}{l}\text { Upper } \\
\text { Bound }\end{array}$ & Tolerance & VIF \\
\hline \multirow{14}{*}{1} & (Constant) & 3.483 & .377 & & 9.242 & .000 & 2.731 & 4.235 & & \\
\hline & Adequacy of food supply & -.252 & .080 & -.205 & -3.129 & .003 & -.412 & -.091 & .432 & 2.315 \\
\hline & personal hygene of cafeteria workers & .132 & .059 & .113 & 2.240 & .028 & .014 & .249 & .733 & 1.363 \\
\hline & Tc. disagree & .071 & .186 & .026 & .383 & .003 & .299 & .441 & .401 & 2.493 \\
\hline & Tc. strong disagree & -.079 & .118 & -.067 & -.665 & .008 & .315 & .157 & .182 & 5.508 \\
\hline & Tc. neutral & .037 & .074 & .027 & .505 & .005 & .111 & .186 & .645 & 1.550 \\
\hline & typhus & .196 & .115 & .170 & 1.703 & .013 & .034 & .425 & .186 & 5.383 \\
\hline & gastric & .720 & .270 & .264 & 2.667 & .010 & .181 & 1.259 & .189 & 5.280 \\
\hline & cg. strong agree & -.198 & .332 & -.130 & -.595 & .664 & -.860 & .465 & .039 & 5.894 \\
\hline & cg. agree & -.112 & .147 & -.095 & -.761 & .449 & -.405 & .181 & .118 & 8.488 \\
\hline & Cs. high & 1.227 & .156 & -1.037 & -7.840 & .000 & -1.539 & -.915 & .106 & 2.447 \\
\hline & cs. medium & .072 & .082 & -.062 & -.883 & .001 & .235 & .091 & .378 & 2.649 \\
\hline & fd. high & .761 & .309 & .661 & 2.461 & .016 & .144 & 1.377 & .026 & 2.936 \\
\hline & fd. medium & .492 & .283 & .426 & 1.742 & .026 & .072 & 1.056 & .031 & 2.273 \\
\hline
\end{tabular}

Variables such as adequacy of food supply, personal hygiene of cafeteria workers, time of cafeteria agree, time of cafeteria disagree, time of cafeteria strong disagree, time of cafeteria neutral, typhus, gastric, cafeteria sanitation high, cafeteria sanitation medium, food sanitation high, food sanitation medium are variables which are significant whereas cg. strongdis agree, cgagree, cgdisagree, cgneutral are variables which are not significant.

The fitted regression is as follows

$$
=+++\mathrm{X}_{3}+\mathrm{X}_{4}+\mathrm{X}_{5}+\mathrm{X}_{6}+\mathrm{X}_{7}+\mathrm{X}_{8}+\mathrm{X}_{9}+\mathrm{X}_{10+} \mathrm{X}_{11}+
$$

Where, - estimated value of the students academic achievement

adequacy of food supply

$=$ personal hygiene of cafeteria workers

$\mathrm{X}_{3}=$ time of cafeteria agree (tc. agree)

$\mathrm{X}_{4}=$ time of cafeteria disagree (tc. disagree)

$\mathrm{X}_{5}=$ time of cafeteria strong disagree (tc. strong disagree)

$\mathrm{X}_{6}=$ time of cafeteria agree (tc. neutral)

$=$ typhus

$=$ gastric

$\mathrm{X}_{9}=$ cafeteria sanitation (cs. medium)
$\mathrm{X}_{10}=$ cafeteria sanitation (cs. high)

$=$ food sanitation (fd. high)

$=$ food sanitation ( $\mathrm{fd}$. medium)

$$
0.72 \mathrm{X}_{8}+1.227 \mathrm{X}_{9}+0.072 \mathrm{X}_{10}+0.761 \mathrm{X}_{11}+0.492 \mathrm{X}_{12}
$$

\section{Interpretation}

For $=-0.267$ the change in the average value of $\mathrm{Y}$ (the academic achievement of students), is decreased by $26.7 \%$ for students who get adequate food supply when its compared to who didn't get adequate food supply holding the other variables as constant.

For $=0.132$ this value is positive, mean that there is direct relationship between students who say yes and academic achievement of student (our reference "no"), this means academic achievement of students is increased by $13.2 \%$ as compared as students who say no. Therefore personal hygiene of cafeteria workers has significant impact on academic achievement of students'.

$=-0.2$ this value is negative, mean that there is inverse relationship between students who say tc. agree and academic achievement of students (our reference"strong agree"), this means academic achievement of students is 
decreased by $20 \%$ as compared as strong agree. This implies students who say tc. agree is less likely than students who say strong agree. Therefore time of cafeteria have significant impact on academic achievement of students'. For other categories of this variable interpret in the same way.

For $=0.196$ this implies there is direct relationship between typhus and the academic achievement of students (reference 'typhoid'). This means the change in the average value of $\mathrm{Y}$ (the academic achievement of students) is increased by $19.6 \%$ as compared as typhoid patient students.

For $=0.72$ when we compare gastric with typhoid the change in the average value of Y (the academic achievement of students) is increased by $7.2 \%$ because this value is positive. Therefore health related problem have significant impact on the academic achievement of students.

For $=1.227$ the value of is positive, this means by $12.27 \%$ academic achievement of students is decreased by $12.27 \%$ as compared as the students who say low (our reference low). This implies cafeteria sanitation has significant impact on academic achievement of students.

For $=0.072$ the value of is positive, this means academic achievement of students is decreased by $7.2 \%$ as compared as low (our reference low). This implies cafeteria sanitation has significant impact on academic achievement of students.

For $=0.761$ when we compare fd. high with fd. Low (reference) the change in the average value of $\mathrm{Y}$ (the academic achievement of students) is increased by $76.1 \%(0.761$ times $)$, because this value is positive. Therefore food sanitation has significant impact on the academic achievement of students.

For $=0.492$ the value of is positive, mean that there is direct relation ship between students who say fd. medium and low academic achievement of students students (our reference'low'), this means academic achievement of students is increased by $49.2 \%$ as compared as low. This implies food sanitation has significant impact on academic achievement of students.

\section{Conclusion}

This study investigates the impacts of food related factors that affect the student's academic achievement in Debre Berhan University. Based on the findings of the study, the following conclusions are drawn as major impacts of food related problems on the student's academic achievement in the university. From the output or result of descriptive and inferential statistic the following conclusions obtained.

1. From the above frequency table and the analysis of multiple linear regression the academic achievement of students was mostly affected by adequacy of food supply, quality of cafeteria sanitation, health related problem, food sanitation and personal hygiene of cafeteria workers, time of cafeteria and those factors decreases the academic achievement of students from time to time.

2. From the empirical results it can be conclude that the variables such as adequacy of food supply, quality of cafeteria sanitation, food sanitation, health related problem, personal hygiene of cafeteria workers and time of cafeteria are determinant factors that leads the students good result.

\section{References}

[1] WFO (2018). International world food Organization; food problem that exist in developing countries.

[2] Tilahun. Woldu. (2011). Status meaning that maintain life and physical, cognitive and social development, Research of Dilla University.

[3] Slack, (1999). According to an acceptable definition food security is to access all people times to enough food for living with an active healthy life.

[4] FAO, WHO, WFP. (2018), The state of food security and Nutrition

[5] Frongillo, E. A., Joni, D. F., \& Jones, S. J. (2006). Food stamp program participation for better academic learning among school children. The Journal of Nutrition, 136, 1077-1080. Retrieved from http://jn.nutrition.org.

[6] Bloom, A. (2009, May 22). Revealed: Fast food diet can result in slow-brain children. Times Educational Supplement, pp. N\&O8.

[7] Kretchmer, N., Beard, J. L., Carlson, S. (2016). The role of nutrition in the development of normal cognition. The American Journal of Clinical Nutrition (63), 997S-1001S. Retrieved from http://www.ajen.org.

[8] Taras, H. \& Potts-Datema, W. (2005). Obesity and student performance. The Journal of School Health, 75 (8), 291-295.

[9] Dauncey, M. J. (2009). New insights in to nutrition and cognitive neuroscience. Proceedings of the Nutrition Society (68), 408-415. doi: $10.1017 / \mathrm{S} 0029665109990188$.

[10] Gomez-Pinilla, F. (2008). Brain foods: The effects of nutrients on brain function. National Review of Neuroscience, 9 (7), 568-578. doi: $10.1038 / \mathrm{nrn} 2421$.

[11] Jager, C. A. \& Kovatcheva, A. (2010). Summary and discussion: Methodologies to assess long-term effects of nutrition on brain function. Nutrition Reviews, 68 (suppl. 1), S53-S58. doi: 10.1111/j.1753-4887.2010.00332.

[12] Smith, A. P. (2010). An investigation of the effects of breakfast cereals on alertness, cognitive function, and other aspects of the reported well-being of children. Nutritional Neuroscience, 5 (13), 230-236. doi: 10.1179/147683010X12611460764642.

[13] David, J. L. (2010). What research says about school meals and learning. Educational Leadership, 88-89. Retrieved from http://www.ascd.org.

[14] Hansen. J. (2000), academic achievements of students depends on socio economic factors.

[15] Cochran, W. G. (1977). Sampling Techniques, 3rd, Ed, John Wiley\& Sons, Inc., New York. 